\title{
La Investigación ambiental para el Conurbano Bonaerense
}

\author{
Miguel A. Blesa \\ Instituto de Investigación e Ingeniería Ambiental, Universidad Nacional de San Martín. \\ E-mail: MBlesa@unsam.edu.ar
}

\section{Resumen}

El conurbano bonaerense, con una población de alrededor de 10 millones de habitantes, está recién comenzando a desarrollar tareas de investigación y desarrollo. Este desarrollo tiene como apoyatura principal las universidades de gestión pública y de gestión privada instaladas en el territorio que se han ido creando en su mayoría en los últimos 25 años. Por contrapartida, las instituciones emblemáticas de la ciencia en la nación (el Consejo Nacional de Investigaciones Científicas y Técnicas CONICET) y en la provincia de Buenos Aires (la Comisión de Investigaciones Científicas CICPBA) tienen solo una presencia incipiente en el territorio. En ese contexto institucional se ha comenzado a desarrollar una interesante tarea de investigación y desarrollo, y están dadas las condiciones para avanzar fuertemente en la construcción de un espacio acorde con las necesidades de la región en el siglo XXI. Para orientar prioridades, es interesante recurrir como marco de referencia al documento de la Secretaría de Planeamiento y Políticas del ex Ministerio de Ciencia, Tecnología e Innovación Productiva, Identificación de las problemáticas centrales de la Argentina para alcanzar un desarrollo sustentable. Sugerencias para superarlas desde el ámbito de incumbencia del Ministerio de Ciencia, Tecnología e Innovación Productiva. Programa CITIDES, Junio de 2017. Se pone allí el foco en los Objetivos del Desarrollo Sustentable de Naciones Unidas, para identificar temas y formas de hacer investigación: multidisciplinaria, en forma de grandes proyectos integradores, en red, con participación de los usuarios potenciales (gobierno, comunidad, empresas) y con objetivos bien definidos. En síntesis, para orientar nuestras prioridades en investigación, cabe pensar en un gran programa que apunte a contestar la pregunta: ¿En qué conurbano queremos vivir?

Palabras clave: investigación científica, conurbano bonaerense, redes de investigación

\section{1- Protección ambiental y desarrollo sustentable}

El informe Nuestro Futuro Común elaborado por la Comisión Mundial sobre el Medio Ambiente y el Desarrollo en 1987 define al desarrollo sostenible como "la satisfacción de las necesidades de la generación presente sin comprometer la capacidad de las generaciones futuras para satisfacer sus propias necesidades".

\footnotetext{
${ }^{1}$ Organización de las Naciones Unidas (1987). Informe de la Comisión Mundial sobre el Medio Ambiente y el Desarrollo. "Nuestro futuro común". Recuperado de http://www.un.org/es/ga/president/65/issues/ sustdev.shtml
}

El desarrollo sustentable asocia el desarrollo -un proceso de cambio para el mejoramiento de las condiciones de vida, el bienestar de los individuos y la sociedad en general-, y la sustentabilidad -la capacidad de mantener en el tiempo determinada situación 0 condición.

Es así que a veces se pone más el foco en la conservación ambiental y a veces en el crecimiento económico, pero en esencia el desarrollo sustentable debe responder al desafío formulado en el interrogante: ¿cómo promover y mejorar el bienestar de las mayorías distribuyendo la riqueza y los 
costos y beneficios ambientales y sociales de manera justa, preservando la naturaleza que a su vez es parte proveedora y necesaria de ese bienestar?

Se debe entonces adoptar una concepción integral del desarrollo que involucre múltiples dimensiones: la ambiental, la sanitaria, la social, la cultural, la educativa, la científico-tecnológica, la ética, la política, entre otras; un futuro sustentable sólo será posible si estas otras dimensiones del desarrollo reciben la misma prioridad que la económica.

Para la Organización de las Naciones Unidas el desarrollo sustentable tiene tres pilares que deben ser contemplados de manera equilibrada: el desarrollo económico, el desarrollo social y la protección del medio ambiente. La dimensión ambiental experimenta presiones negativas derivadas del crecimiento económico basado en un consumo de recursos naturales sin precedentes. El uso de tecnologías más adecuadas y el uso racional de los recursos buscan desacoplar la extracción de recursos naturales y el crecimiento económico. Sin embargo, estas mejoras no han logrado contrarrestar el incremento combinado de la población y el consumo per cápita de bienes y servicios².

\footnotetext{
2 Intergovernmental Panel on Climate Change (2014). Drivers, Trends and Mitigation. Contribution of Working Group III to the Fifth Assessment Report of the Intergovernmental Panel on Climate Change. Recuperado de https://www.ipcc.ch/pdf/assessment-report/ar5/wg3/ipcc_wg3 ar5_chapter5.pdf
}
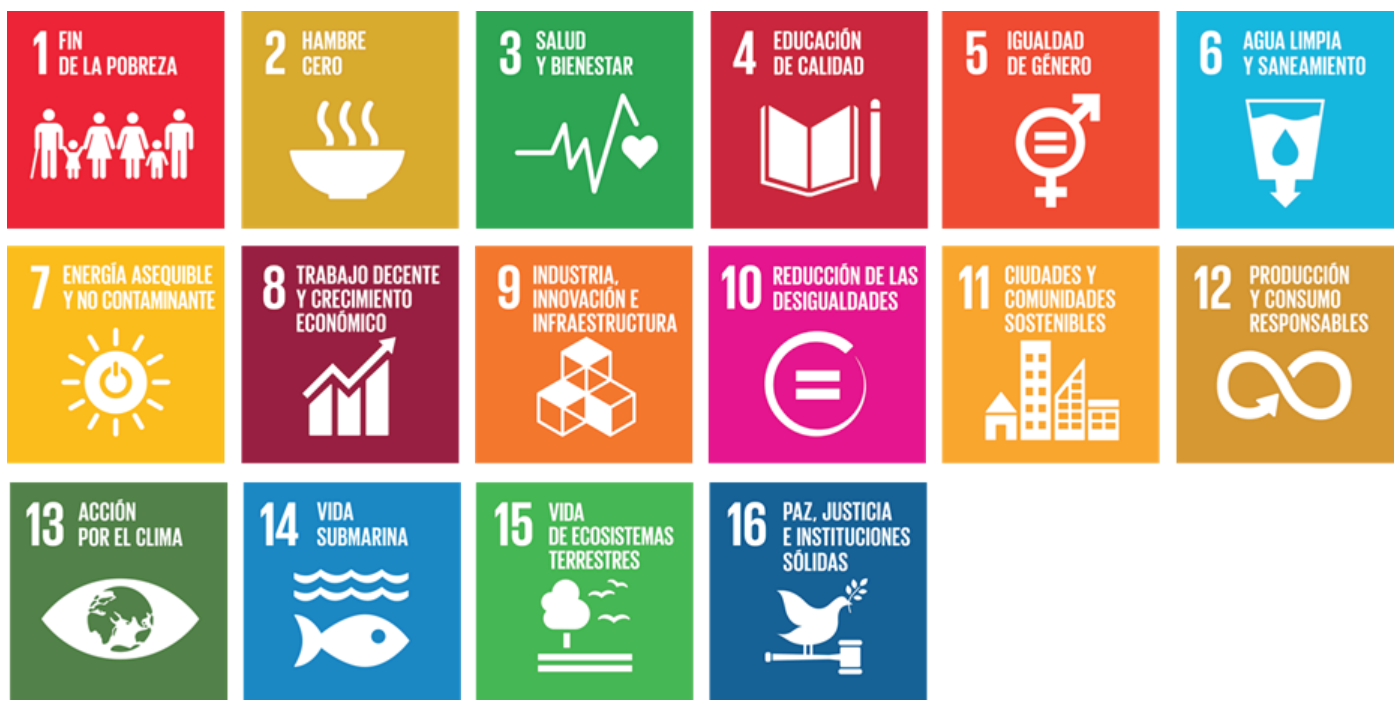

${ }^{3}$ Véase la evolución del índice de Gini que mide la desigualdad según los datos del World Bank Group: https://datos.bancomundial.org/indicador/SI.POV.GINI

${ }^{4}$ Organización de las Naciones Unidas (2015). Objetivos de Desarrollo Sustentable. Recuperado de http://www.un.org/sustainabledevelopment/es/objetivos-de-desarrollo-sostenible/

La importancia de superar visiones reduccionistas y sesgadas del desarrollo está siendo de algún modo reconocida a nivel internacional. Desde la Cumbre de la Tierra de 1992, las Naciones Unidas, en representación de los países miembros, se ha planteado sumar al Desarrollo Sustentable como su bandera, como así lo demuestran Ios diecisiete Objetivos del Desarrollo Sostenible (ODS) adoptados en 2015 y la diversidad de problemáticas que éstos abarcan. Los ODS que se proponen alcanzar en el período 2015-2030 son los siguientes ${ }^{4}$ (Fig.1)
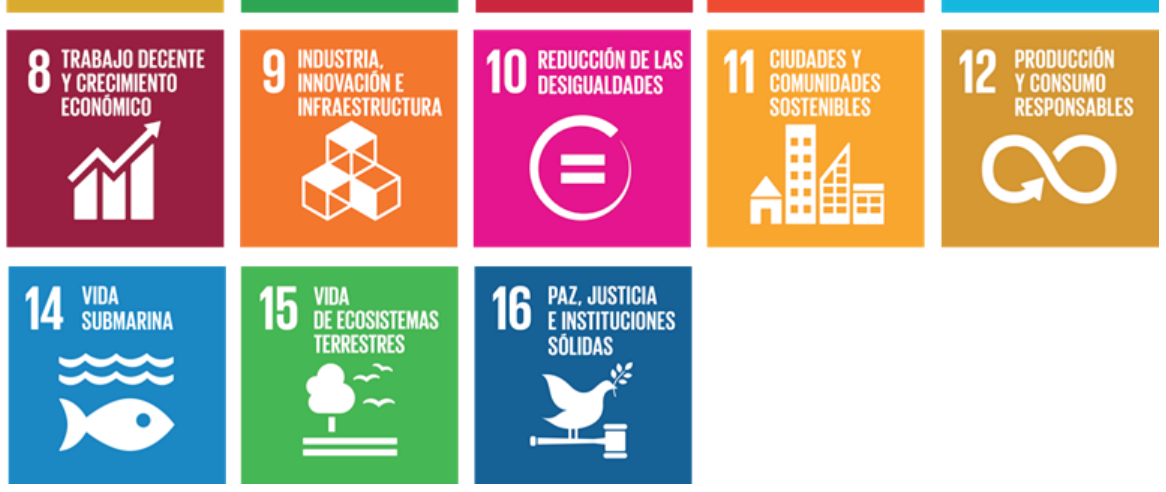

Figura 1. Objetivos del Desarrollo Sostenible (ODS).

Fuente: Organización de las Naciones Unidas (2015). 
Los ODS entraron en vigor oficialmente el $1^{0}$ de enero de 2016. Con estos nuevos objetivos de aplicación universal se espera que los países intensifiquen los esfuerzos para poner fin a la pobreza en todas sus formas, reducir la desigualdad y luchar contra el cambio climático, garantizando, al mismo tiempo, que nadie quede al margen de los beneficios que esta nueva forma de desarrollo genere.

La Argentina, como parte de la comunidad internacional, también adoptó estos objetivos de desarrollo sustentable. Es en este marco, pero también por propia necesidad, que la Argentina debe identificar las problemáticas que hacen que su desarrollo no encuentre estabilidad económica, exhiba profundas desigualdades sociales, e impacte negativamente y de manera insostenible sobre nuestro capital natural. Son estas problemáticas las que el país deberá enfrentar si quiere converger hacia un sendero de desarrollo que integre de manera armónica cada una de las dimensiones que hacen a su propia sustentabilidad.

¿Cuáles son las principales problemáticas del desarrollo sustentable de relevancia para la Argentina? La Comisión Asesora de Desarrollo Sustentable (CADES) del Ministerio de Ciencia Tecnología e Innovación Productiva (MINCyT) las ha identificado, agrupándolas en complejos causales. ${ }^{5}$ Para ello identificó aquellos nodos problemáticos esenciales que denotan los principales obstáculos, pero también oportunidades, para el desarrollo social, económico y ambientalmente sustentable de la Argentina, y los integró en complejos multidimensionales, y por ende transversales a los sectores en los que habitualmente se divide la actividad de un país, e incluso transversales a las diferentes áreas de conocimiento y las diferentes ramas de las ciencias. Los complejos causales agrupan factores asociados causalmente que facilitan la identificación de acciones, políticas y estrategias apropiadas, y favorecen la comprensión de los diferentes actores involucrados.

El proceso de identificación de los complejos prioritarios ha tenido en consideración algunos criterios mínimos. En primer lugar, su pertenencia a la problemática del desarrollo

\footnotetext{
${ }^{5}$ Secretaría de Planeamiento y Políticas en Ciencia, tecnología e Innovación Productiva (2017). Identificación de las problemáticas centrales de la Argentina para alcanzar un desarrollo sustentable. Sugerencias para superarlas desde el ámbito de incumbencia del Ministerio de Ciencia, Tecnología e Innovación Productiva.
}

sustentable como tal, y no sólo a la problemática del desarrollo 0 a la problemática ambiental. En segundo lugar, su importancia ambiental y socioeconómica para la Argentina específicamente, lo que hace que no necesariamente los complejos temáticos repliquen de manera exacta los ODS definidos universalmente aunque sí exista una relación con ellos. Y finalmente, la priorización de los complejos causales ha tenido en cuenta su grado de contacto con la ciencia y la tecnología.

De este modo se han identificado los siguientes complejos causales que cubren los nodos más problemáticos para el desarrollo sustentable de nuestro país:

\section{Complejo patrones de producción y consumo \\ 2. Complejo ecosistemas y sociedad \\ 3. Complejo servicios \\ 4. Complejo institucional/gobernanza política \\ 5. Complejo pobreza y desigualdad \\ 6. Complejo cambio ambiental global}

\section{2- La ciencia y la tecnología en el Conurbano Bonaerense} Hagamos ahora una digresión para focalizarnos en el conurbano bonaerense. Uso el término conurbano bonaerense de manera laxa, para referirme a esa gran área urbana y semirural que rodea la Ciudad Autónoma de Buenos Aires y que alberga alrededor de 10 millones de habitantes. La ley 13473/06 (junio de 2006) delimita el Conurbano bonaerense en un área territorial comprendida por 33 municipios agrupados en 8 diferentes zonas:

- Zona Sureste, integrada por los partidos de Almirante Brown, Avellaneda, Berazategui, Florencio Varela y Quilmes.

- Zona Sur, integrada por los partidos de Esteban Echeverría, Ezeiza, Presidente Perón y San Vicente.

- Zona Suroeste, integrada por los partidos de La Matanza, Lanús y Lomas de Zamora.

- Zona Oeste, integrada por los partidos de Hurlingham, Ituzaingó, Morón.

- Zona Noroeste, integrada por los partidos de General Rodríguez, Merlo, Moreno y Pilar.

- Zona Norte Centro, integrada por los partidos de General San Martín, José C. Paz, Malvinas Argentinas, San Miguel y Tres de Febrero 
- Zona Noreste, integrada por los partidos de Escobar, San Desde el punto de vista de esta presentación, nos referimos Fernando, San Isidro, Tigre y Vicente López. a ese conglomerado, con la excepción de la Zona Gran La

- Zona Gran La Plata, integrada por los partidos Plata, que tiene características diferentes (Fig.2) de Berisso, Ensenada y La Plata.

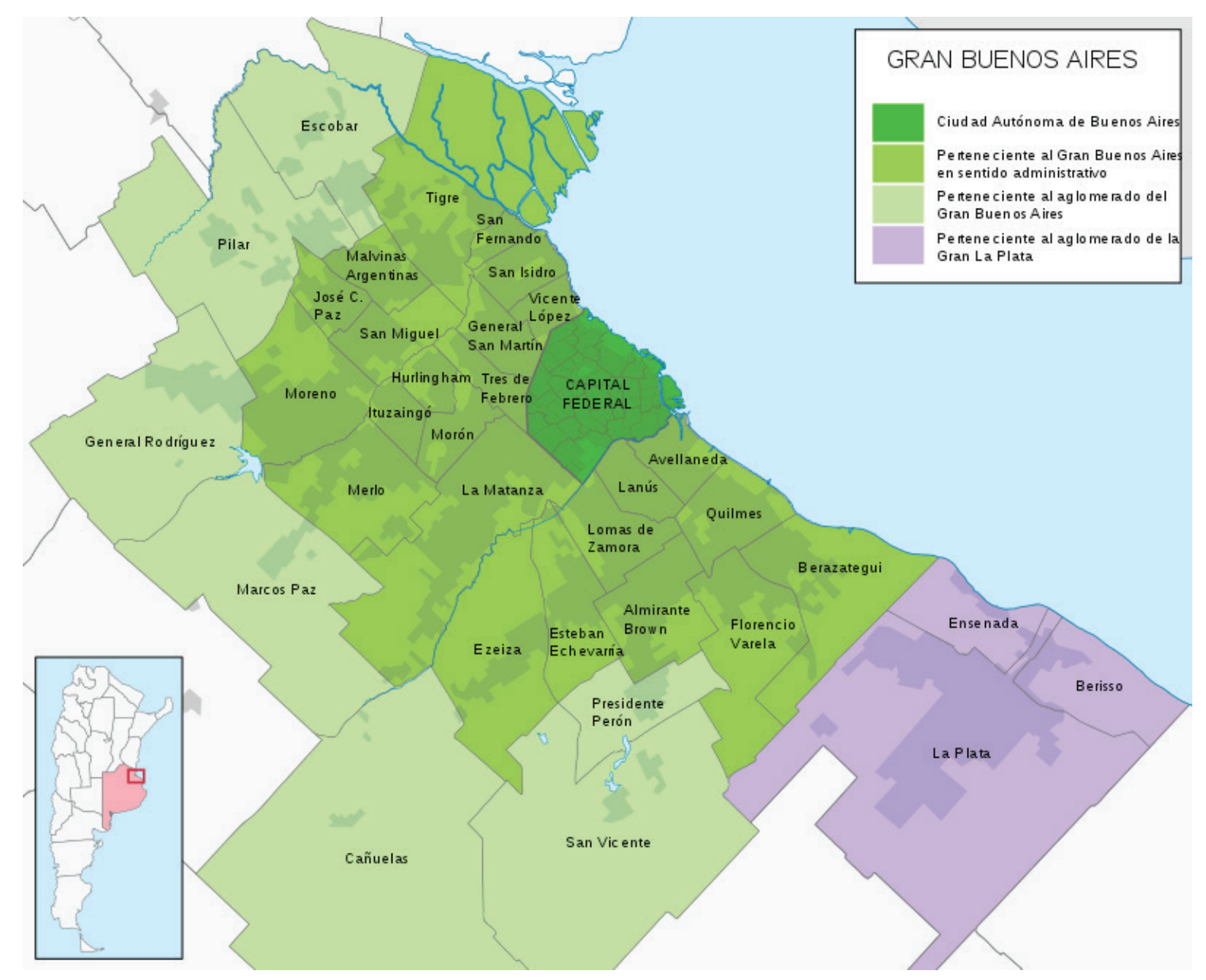

Figura 2. El Gran Buenos Aires.

Fuente: Wikipedia, https://es.wikipedia.org/wiki/Gran_Buenos_Aires

A partir de la creación de universidades en las décadas de 1990, 2000 y 2010, la oferta académica en la región es amplia, como puede apreciarse en las tablas que siguen (Tablas I y II).

La Comisión de Investigaciones Científicas de la Provincia de Buenos Aires tiene 84 Centros (propios y asociados). De ellos, en el conurbano se ubican solo 11: 3 en Lomas de Zamora, 2 en Lanús, 1 en San Martín, 1 en Pilar, 1 en Florencio Varela y 3 en Avellaneda (indicados con flechas en el mapa). Queda claro que hay una ausencia casi total en gran parte del conurbano bonaerense. A diferencia de muchos de los institutos más tradicionales del sistema de ciencia y técnica, tanto provincial como nacional, las temáticas de estos institutos son en general altamente interdisciplinarias, y tienen una mirada hacia el territorio. Cinco de esos institutos están vinculados con temas ambientales y de salud pública. Está claro que recién ahora se está poniendo en el mapa a la investigación científica en el conurbano y queda mucho camino por recorrer. La 


\begin{tabular}{|c|c|}
\hline & la I. UNIVERSIDADES DE GESTIÓN PÚBLICA EN EL CONURBANO (Fecha de creación) \\
\hline & Universidad Tecnológica Nacional (UTN) (Pacheco) (1972) \\
\hline Zona & Universidad Nacional de Gral. Sarmiento (UNGS) (Malvinas Argentinas) (1993) \\
\hline & Universidad Nacional de Gral. San Martín (UNSAM) (San Martín) (1992) \\
\hline Norte & Universidad Nacional de Hurlingham (UNAHUR) (Hurlingham) (2014) \\
\hline & Universidad Nacional de Luján (UNLU) (San Miguel - San Fernando - Campana) (1972) \\
\hline & Universidad Nacional de Avellaneda (UNDAV) (Avellaneda - Piñeyro) (2010) \\
\hline & Universidad Nacional de Lomas de Zamora (UNLZ) (Lomas de Zamora) (1972) \\
\hline Zona & Universidad Tecnológica Nacional (UTN) (Avellaneda) (1954?) \\
\hline & Universidad Provincial de Ezeiza (2009) \\
\hline Sur & Universidad Nacional Arturo Jauretche (Florencio Varela y otras localidades) (2009) \\
\hline & Universidad Nacional de Quilmes (UNQ) (Quilmes) (1989) \\
\hline & Universidad Nacional de Lanús (UNLA) (Lanús) (1995) \\
\hline & Universidad Tecnológica Nacional (UTN) (Campana - Haedo) \\
\hline & Universidad Nacional de La Matanza (UNLAM) (La Matanza) (1989) \\
\hline Zona & Universidad Nacional de Moreno (Moreno) (2009) \\
\hline 0este & Universidad Nacional José C. Paz (José C. Paz) (2009) \\
\hline & Universidad Nacional del Oeste (Merlo) (2009) \\
\hline & Universidad Nacional de Tres de Febrero (UNTREF) (Tres de Febrero) (1995) \\
\hline
\end{tabular}

Fuente: Consejo Interuniversitario Nacional http://www.cin.edu.ar/instituciones-universitarias/. Fechas de creación: elaboración propia; ver en especial Accinelli, A. y Macri, A. La creación de las universidades nacionales del conurbano bonaerense: Análisis comparado de dos períodos fundacionales. Revista Argentina de Educación Superior ISSN 1852-8171 / Año 7/ Número 11 / noviembre 2015. Recuperado de https://www.uai.edu.ar/media/42455/ganadores-2016_la-creaci\%C3\%B3n-de-lasuniversidades-del-conurbano-bonaerense.pdf

comparación del número de institutos con la densidad poblacional es clara.

El contraste entre población y unidades ejecutoras de CONICET, ahora a nivel nacional, es aún más marcado. Sobre 266 Unidades Ejecutoras, solo pude encontrar 7 en el conurbano (2,6\%), ${ }^{6}$ altamente concentradas en San Martín, frente a un $25 \%$ de población del país. Seguramente este número se incrementará muy pronto, en vista del rápido crecimiento del número de unidades ejecutoras, pero esto no cambia apreciablemente el panorama.

Una lección fundamental es la importancia de la temática del desarrollo sustentable, y dentro de ella de la temática de

${ }^{6}$ Véase: https://red.conicet.gov.ar/nomina-y-mapa-institucional/
protecciónambiental.Ynoquedadudasquelas peculiaridades del conurbano dan razones más que suficiente para encarar un programa ambicioso y estructurado que involucre a todas las capacidades de la región, especialmente universidades de gestión pública y de gestión privada. La fragmentación y desconexión de los esfuerzos individuales los torna casi estériles. Conozco a fondo los ejemplos vinculados con la gestión de los recursos hídricos, en particular cómo encarar la gestión de las aguas arsenicales para consumo humano y para producción agrícola-ganadera.

Parece obvio que están dadas las condiciones para que, sobre la base de las instituciones académicas existentes, se diseñe y se implemente un gran programa de investigación 


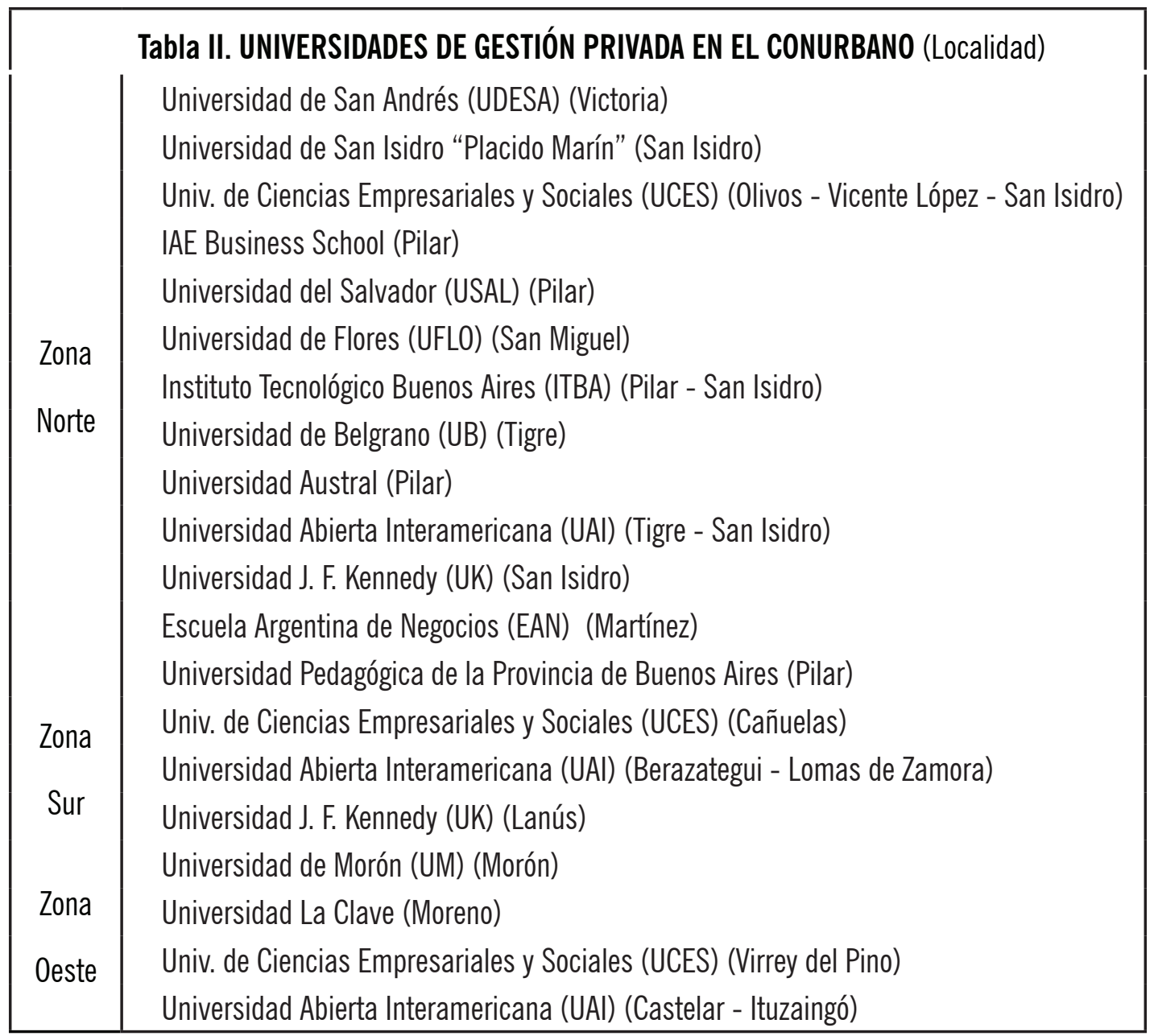

Fuente: https://www.altillo.com/universidades/argentina/universidades_arg_granbuenosaires.asp

que podría llamarse iEn qué conurbano queremos vivir? Existen antecedentes que apuntan en esa dirección. La organización Future Earth está impulsando a nivel internacional un ambicioso programa de investigación transdisciplinario bajo el lema ¿En qué planeta queremos vivir? ${ }^{7}$ Los decanos de las facultades de ciencias sociales de la Argentina construyeron el Programa de Investigaciones sobre la Sociedad Argentina Contemporánea (PISAC). ${ }^{8}$ La Red de Universidades Bonaerenses para el Desarrollo de la región (RUNBO) avanza sobre distintas líneas de acción relacionadas con la información, base de datos y los problemas hidrológicos reconocidos en la Provincia de

${ }^{7}$ Para más información, véase: http://www.futureearth.org/

${ }^{8}$ Véase: http://pisac.mincyt.gob.ar/
Buenos Aires. ${ }^{9} Y$ finalmente, en 2008 siete universidades del conurbano crearon la Red de Universidades Nacionales del Conurbano Bonaerense (RUNCOB), institución a la que se fueron agregando después otras universidades de la región. ${ }^{10}$ Esta red puede adoptar un papel protagónico en la construcción de una gran propuesta regional integradora en investigación y desarrollo.

\section{3- Los complejos causales y la investigación para el desarrollo sustentable}

En ese contexto poblacional y de oferta académica,

${ }^{9}$ Véase: https://www.runbo.unlp.edu.ar/

${ }^{10}$ Véase por ejemplo: http://www.ridaa.unicen.edu.ar/xmlui/hand-

le/123456789/91 
retomemos el documento de la CADES para ver el amplio listado de temas que se agrupan en los complejos causales. He suprimido unos pocos que son de escasa 0 nula relevancia para la región (por ejemplo, pesquerías). Sin embargo, la lista sigue siendo extremadamente amplia como se ve a continuación.

\section{Complejo patrones de producción y consumo}

- Cambios en el uso del suelo vinculados a la oferta y demanda del suelo con distintos fines: agropecuario, urbano, periurbano, industrial y natural, y su integración territorial.

- Enfoque de nexos integrando los procesos de oferta y demanda de recursos de base como energía, agua, suelos y producción de biomasa, en especial alimentos.

- Recursos energéticos y matriz energética incluyendo oferta y demanda de energía, tendencias, escenarios, energías renovables y no renovables, uso racional y eficiente de la energía e indicadores de sustentabilidad energética.

- Desarrollo de recursos endógenos disponibles en diferentes regiones del país, tales como diversos tipos de biomasa, recursos hídricos, geotérmicos, oceánicos, paisajísticos, distintos servicios ecosistémicos.

- Desarrollo de tecnologías locales y fortalecimiento de capacidades existentes, tradicionales y no tradicionales.

- Metabolismo industrial y su funcionamiento en términos de la demanda de recursos, energía, desacople, productividad, residuos y efluentes.

- Metabolismo urbano: Ciudades "energívoras" y ajustes en el flujo físico de materiales y energía, disposición final de residuos y cambios en el uso del suelo.

- Logística y transporte: Análisis de emisiones, flujos y sistemas multimodales. Impactos de la infraestructura y cambios en los sistemas de transporte.

- Recursos no renovables: El papel del reciclado de materiales y los recursos no renovables (elementos extraídos vs. porcentaje y tendencias del reciclado).

- Cadenas de producción y consumo: Oferta y demanda a niveles, local, regional y global, análisis de flujos físicos y económicos, y relaciones monetarias y biofísicas.

- Los sistemas alimentarios, pautas de consumo y nutrición.
- Educación ambiental, comunicación y formación.

- Efectos del sistema económico y financiero y su relación con los recursos naturales.

- Complejidad ambiental y hábitos de consumo: Límites físicos del sistema económico.

\section{Complejo ecosistemas y sociedad}

- Servicios ecosistémicos relacionados con la producción de bienes y servicios materiales en forma directa 0 indirecta (por ejemplo: polinización, descomposición de materia orgánica, retención de suelos, regulación del clima y el agua), como así también bienes inmateriales como el sentido de pertenencia, la recreación, el goce estético y otros servicios culturales.

- Recursos energéticos y matriz energética, en términos de la oferta y demanda, tendencias, escenarios, energías renovables y no renovables, uso justo y eficiente de la energía e indicadores de sustentabilidad energética.

- Uso del suelo y cambios en el uso del suelo.

- Uso del agua y reservorios de agua dulce: protección y restablecimiento de ecosistemas relacionados con la regulación del flujo y calidad química y biológica del agua, incluidos los bosques, las montañas, los humedales, los ríos, los acuíferos y los lagos.

- Gestión integrada de recursos hídricos (a todos los niveles, incluso mediantela cooperacióntransfronteriza), considerando cantidad y calidad, aguas superficiales y aguas subterráneas, tratamientos, saneamiento ambiental, control de desechos industriales. Tecnologías de bajo costo y fácil aplicación de acuerdo a la necesidad de cada zona. Presencia de arsénico en agua de consumo con consecuencias directas en salud humana.

- Biodiversidad en sistemas naturales, seminaturales, agrícolas y urbanos.

- Planificación y ordenamiento territorial.

- Procesos de urbanización y metabolismo urbano.

- Vínculos económicos, sociales y ambientales positivos entre las zonas urbanas, periurbanas y rurales mediante el fortalecimiento de la planificación del desarrollo nacional y regional. 


\section{Complejo servicios}

- Servicios urbanos: agua, saneamiento, residuos, gas, energía, salud, espacios verdes, educación, transporte público, esparcimiento cultural, deportivos y otros.

- Desarrollo de cadena de proveedores, formación técnica, fortalecimiento institucional, capacitación y concientización.

- Asentamientos humanos: urbanización inclusiva y sostenible, y la capacidad para una planificación y gestión participativas, integradas y sostenibles de los asentamientos humanos (en el caso de poblaciones dispersas, la posibilidad de utilizar energías renovables distribuidas tiene una gran potencialidad y se encuentra muy poco desarrollada).

- Calidad del aire y contaminación sonora y visual.

- Educación formal e informal, ciencia y tecnología.

\section{Complejo institucional/gobernanza política}

- Arreglos institucionales para el desarrollo sustentable.

- Marcos regulatorios que promuevan la provisión de los servicios de modo seguro y sustentable, promoviendo el uso justo y eficiente de los recursos energéticos.

- Desarrollo de herramientas que permitan a los usuarios de los senvicios elegir racionalmente los más convenientes y eficientes, por ejemplo, a través de sistemas de etiquetado de eficiencia y calidad de artefactos domésticos y viviendas.

- Derecho ambiental y legislación ambiental.

- Derechos materiales e intelectuales sobre el patrimonio ambiental, incluyendo la biodiversidad.

- Relación provincias/recursos naturales (Art.41 de la Constitución Nacional).

- Participación ciudadana y modelos de participación en la administración de bienes de acceso común y privado.

- Modos de producción de conocimiento y acceso al conocimiento como modo de control del desempeño de las instituciones.

- Redes de producción y consumo que fortalezcan la soberanía alimentaria.

- Sistema financiero y sistema impositivo que promueva los modos más sustentables de construcción de viviendas y la adquisición de productos eficientes.

- Integración de los recursos naturales, la biodiversidad y los servicios ecosistémicos en la economía y la legislación.

- Herramientas de análisis multidimensionales para el proceso de toma de decisión.

- Indicadores de desarrollo sustentable.

- Concientización ciudadana: Desarrollo de programas educativos e informativos a nivel de las escuelas y de los medios de difusión masiva, que promuevan la elección por parte de los ciudadanos de productos y servicios seguros y eficientes energéticamente.

- Conflictos socio-ambientales por acceso a la tierra, los cuerpos de agua (incluidos los recursos del mar) y los beneficios sociales de la diversidad.

- Alianzas internacionales y rol en foros internacionales.

\section{Complejo pobreza y desigualdad}

- Nutrición y soberanía alimentaria.

- Formación y capacitación.

- Acceso a servicios urbanos: vivienda, salud, educación, agua y saneamiento, energía, residuos, espacios verdes y esparcimiento.

- Desarrollo de modos sustentables de uso de la energía en regiones de poblaciones dispersas, adecuadas a las características geográficas, ambientales y sociales.

- Apoyo a vínculos sociales y ambientales positivos entre zonas urbanas, periurbanas y rurales.

- Intervención en el mercado del suelo para generar opciones de tierra y vivienda para sectores populares.

- Desarrollo de tecnologías apropiadas.

\section{Complejo cambio ambiental global}

- Medición y observación de variables climáticas.

- Predicciones y proyecciones climáticas.

- Eventos extremos: Olas de calor, inundaciones, sequías. Entendimiento, monitoreo y prevención.

- Sistemas de alerta temprana.

- Manejo de la gestión de riesgos de desastres.

- Relación entre las ciudades y el cambio climático.

- Planeamiento territorial, urbano y rural.

- Manejo de costas.

- Planes de mitigación/adaptación a diferentes niveles: Municipal, provincial, nacional.

- Matriz energética primaria y eléctrica, y uso racional y 
eficiente de los recursos energéticos.

- Sistemas de transporte: Público y de carga, transporte particular, transporte multimodal.

- Manejo sustentable de la producción agrícola y ganadera.

- Manejo y conservación de los ecosistemas terrestres (por ejemplo: bosques, humedales) ante un contexto de impactos conjuntos de cambios climáticos y de uso de la tierra. comercial, industrial.

- Manejo de residuos urbanos e industriales.

La metodología de identificación de complejos causales conduce a que ciertos temas toman relevancia en varios, cuando no en todos los complejos causales. Tomemos dos ejemplos: energía y agua. Se muestran a continuación los temas de interés de cada uno de los complejos causales en los cuales esos temas aparecen (Figs. 3 y 4).

- Eficiencia energética del lado del consumo: Residencial,

\section{La energía en los distintos complejos (I)}

Complejo patrones de producción y consumo

- Enfoque de nexos integrando los
procesos de oferta y demanda de
recursos de base como energia,
agua, suelos y producción de
biomasa, en especial alimentos.
- Recursos energéticos y matriz
energética incluyendo ofertay
demanda de energía, tendencias,
escenarios, energias renovablesy
no renovables, uso racionaly
eficiente de la energia e
indicadores de sustentabilidad
energética.
- Metabolismourbano: Ciudades
"energivoras"y ajustes enelflujo
físico de materiales y energia,
disposición final de residuos y
cambios en el uso del suelo.

- Enfoque de nexos integrandolos procesos de ofertay demanda de recursos de base como energia, agua, suelos y producción de biomasa, en especial alimentos.

Recursos energéticos y matriz escenarios, energias renovablesy no renovables, uso racionaly energética.

- Metabolismourbano: Ciudades fisico de materiales y energia, cambios en el uso del suelo.

\section{Complejo ecosistemas y sociedad}

- Recursos energéticosy
matriz energética, en
términos de la ofertay
demanda, tendencias,
escenarios, energias
renovables y no
renovables, uso justo y
eficiente de la energia e
indicadores de
sustentabilidad
energética.

- Servicios urbanos: agua, saneamiento, residuos, gas, energia, salud, espacios verdes, educación, transporte público, esparcimiento cultural, deportivosy otros.

\section{La energía en los distintos complejos (II)}

Complejo pobreza y desigualdad

\footnotetext{
- Acceso a servicios urbanos: vivienda, salud, educación, aguay saneamiento, energía, residuos, espacios verdes y esparcimiento.

- Desarrollo de modos sustentables de uso de la energía en regiones de poblaciones dispersas, adecuadas a las características geográficas, ambientales y sociales.

- Desarrollo de tecnologías apropiadas.
}

\section{Complejo cambio ambiental global}

- Matriz energética
primariay eléctrica, y
uso racionaly
eficiente de los
recursos energéticos.
- Eficiencia energética
del lado del
consumo:
Residencial,
comercial, industrial.

\section{Complejo institucional/ gobernanza política}

Figura 3. Complejos causales: la energía en distintos complejos. 


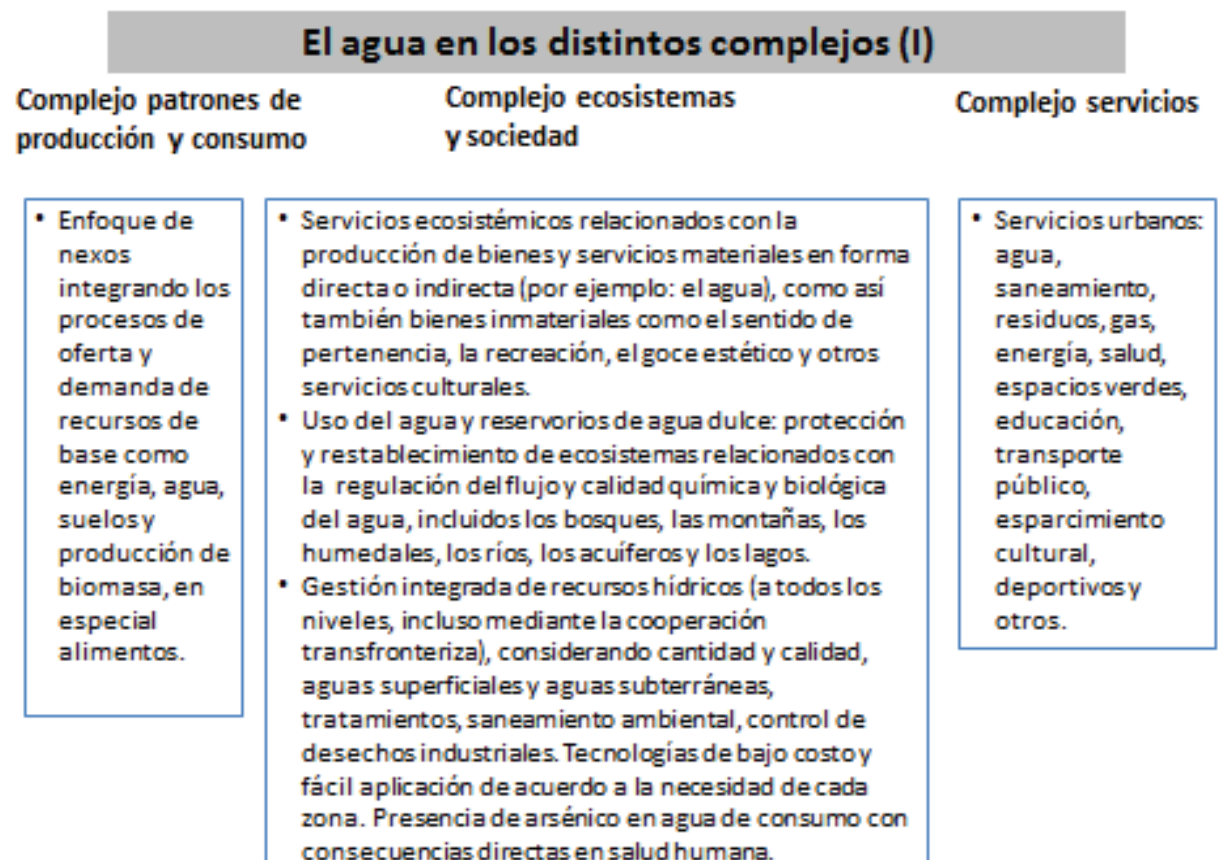

\begin{tabular}{|c|c|c|}
\hline \multicolumn{3}{|c|}{ El agua en los distintos complejos (II) } \\
\hline $\begin{array}{l}\text { Complejo pobreza y } \\
\text { desigualdad }\end{array}$ & $\begin{array}{l}\text { Complejo cambio } \\
\text { ambiental global }\end{array}$ & $\begin{array}{l}\text { Complejo institucional/ } \\
\text { gobernanza política }\end{array}$ \\
\hline $\begin{array}{l}\text { - Acceso a servicios } \\
\text { urbanos: vivienda, salud, } \\
\text { educación, aguay } \\
\text { saneamiento, energía, } \\
\text { residuos, espacios verdes } \\
\text { y esparcimiento. } \\
\text { - Desarrollo de tecnologías }\end{array}$ & $\begin{array}{l}\text { - Eventos extremos: Olas } \\
\text { de calor, inundaciones, } \\
\text { sequias. Entendimiento, } \\
\text { monitoreo y prevención. } \\
\text { - Sistemas de alerta } \\
\text { temprana. } \\
\text { - Manejo de la gestión de }\end{array}$ & $\begin{array}{l}\text { - Marcos regulatorios que } \\
\text { promuevan la provisión de } \\
\text { los servicios de modo } \\
\text { seguro y sustentable, } \\
\text { promoviendo el uso justo y } \\
\text { eficiente de los recursos } \\
\text { energéticos. }\end{array}$ \\
\hline oniadas & $\begin{array}{l}\text { riesgos de desastres. } \\
\text { Relación entre las } \\
\text { ciudades y el cambio } \\
\text { climático. }\end{array}$ & \\
\hline
\end{tabular}

Figura 4. Complejos causales: el agua en distintos complejos.

Es más, a nivel mundial los enfoques integrados de los proyectos de investigación identifican por ejemplo como prioritarios, los estudios de los nexos entre diversos factores. Uno muy conocido es el nexo agua-energíaalimentos. Un estudio integrado de este nexo obliga a pensar de qué forma los cambios que se esperan en el futuro en cada uno de esos factores afectará a los demás. Esos cambios están vinculados por un lado a los factores de crecimiento de la economía, del crecimiento poblacional, y también a la drástica influencia que se cree tendrá el cambio climático global sobre la disponibilidad de agua, de alimentos y también de la energía (por ejemplo de la oferta de hidroelectricidad).

El documento de la CADES describe cómo deben entonces formularse proyectos de I\&D que tengan posibilidad de impacto real. A modo de ejemplo, a continuación se mencionan algunos tipos de contribución que la ciencia y la tecnología podrían hacer para que el crecimiento del país se transforme en desarrollo sustentable, y en particular respete la dimensión ambiental: 
- Identificación dentro de los complejos causales, de los problemas específicos accesibles al enfoque C\&T.

- Relevamiento de la importancia y extensión de los problemas a través de sensores de distinto tipo, encuestas, muestreos, etc.

- Diagnóstico y pronóstico utilizando las herramientas de la investigación científica.

- Identificación de puntos críticos de intervención a través de los análisis sistémicos y estratégicos.

- Participación en la resolución de los problemas y aprovechamiento de las oportunidades desarrollando y/o utilizando soluciones tecnológicas, capacitaciones especializadas, investigación-acción, diálogos cienciapolítica, etc.

- Seguimiento (monitoreo) de la evolución de la problemática utilizando los conocimientos científicos.

- Desarrollo de capacidades e incorporación de "la sustentabilidad" en los programas de investigación y desarrollo que se llevan adelante en el país.

Unas palabras finales sobre el tema cambio Climático Global en el conurbano bonaerense. Las convenciones internacionales piden a los países contribuir a la disminución de la emisión de gases de efecto invernadero. Ello impacta sobre las fuentes a usar para energía, y en los esfuerzos para su uso racional. Esas son acciones de mitigación que influirán sobre el devenir diario de todo el conurbano, pero cuya implementación excede a la gobernanza local. En cambio, son cruciales también las acciones de adaptación al inevitable cambio climático. Esto significa, en los municipios, disponer de mecanismos para enfrentar los eventos climáticos extremos que aumentarán en intensidad $y$ en frecuencia. 